\title{
PROCESS AND PARAMETERS AFFECTING DRUG RELEASE PERFORMANCE OF PREPARED CROSS-LINKED ALGINATE HYDROGEL BEADS FOR EZETIMIBE
}

\author{
K. V. RAMANA REDDY*1, M. V. NAGABHUSHANAM ${ }^{2}$ \\ 1Department of Pharmaceutics, Research Scholar, Acharya Nagarjuna University, Guntur, Andhra Pradesh. India 522002, ${ }^{2}$ Department of \\ Pharmaceutics, Hindu College of Pharmacy, Guntur, Andhra Pradesh, India 522002 \\ Email: srikanth.papa@gmail.com \\ Received: 19 Nov 2016 Revised and Accepted: 21 Dec 2016
}

\section{ABSTRACT}

Objective: The objective of this study was to formulate an oral sustained release delivery system of ezetimibe mucoadhesive beads by ionic gelation technique based on sodium alginate used as a hydrophilic carrier in combination with carbopol 934P which acts as a rate modifier.

Methods: Microbeads of ezetimibe were prepared using an easy method of ionotropic gelation by little modification while in addition of drug. The prepared beads were characterised for mean particle size, entrapment efficiency, swelling capacity, and in vitro release. They were also subjected to various studies such as Fourier Transform Infrared Spectrophotometer (FTIR) Spectroscopy for drug polymer reaction, Scanning Electron Microscopy for surface morphology, and Differential Scanning Calorimetric Analysis to determine the physical state of the drug in the beads.

Results: The microspheres of ezetimibe were formulated successfully. The addition of drug concentration gives higher drug loading and higher conc. of $\mathrm{Alcl}_{+3}$ yields small diameter beads and lower drug entrapment. Analysis of the release profiles showed that the data corresponds to zero order release and the diffusion-controlled mechanism as suggested by Higuchi concept.

Conclusion: It can be concluded that beads produced by the sequential method had higher drug entrapment. Beads produced by simultaneous yields larger beads in diameter. The concept was cleared that drug release was dependent upon the quantity of polymer and increase in conc. of aluminium chloride retarded the drug release in the sequential method. Prepared beads enhance the dissolution of ezetimibe and the oral bioavailability and also reduce the fluctuations in the oral bioavailability.

Keywords: Ezetimibe, Sodium alginate, Ionic gelation, Mucoadhesive microspheres

(C) 2017 The Authors. Published by Innovare Academic Sciences Pvt Ltd. This is an open access article under the CC BY license (http://creativecommons.org/licenses/by/4. 0/ DOI: http://dx.doi.org/10.22159/ijpps.2017v9i2.16235

\section{INTRODUCTION}

Controlled release systems have been extensively developed and advised to superior due to their extensive therapeutic advantages over conventional forms [1]. The current article was aimed to develop a sustained release dosage form of ezetimibe using a natural, biodegradable polymeric carrier sodium alginate for oral dosage form. The versatility of polymeric materials allows ease fabrication of the drug delivery devices with a desirable degree of swelling and consequently of the release of the drug. This microparticulate material as in the form of microbeads may be dispensed as filled in hard gelatin capsules or they can be compressed into a suitable dosage form like tablets or hard gelatin capsule [2]. The principle of gelation or crosslinking of sodium alginate with aluminium chloride is based on the formation of a tight junction between the guluronic acid residues. The gelation and cross-linking are due to the stacking of the glucuronic acid G blocks of alginate chains with the formation of egg-box junction [3]. The numbers of the apparent cross-linking sites within the formed aluminium alginate gel beads increased with increasing sodium alginate concentration in the formulation. This increase in the apparent crosslinking density delayed the alginate gel disintegration in phosphate buffer due to the retardation of $\mathrm{Al}_{+3}$ exchange with $\mathrm{Na}^{+}$and eventually increasing lag time. Increased alginate gel density per unit volume was also thought to affect the decreased pore size within the gels, and thus ezetimibe release becomes slow.

Sodium alginate, the hydrophilic biodegradable polymer obtained from seaweeds has been found to be highly promising with respect to drug delivery because of its high biological safety [4]. Alginate is commonly employed as cross-linked matrices or coats in drug delivery systems [5]. Over the past two decades, pharmaceutical scientists have shown an increased interest in using alginates as biopolymer in the development of drug delivery systems, due to its hydrogel-forming properties [6, 7]. Sustained release drug delivery system using calcium alginate beads $[8,9]$. Sodium alginate has been used as a matrix material for many decades as a coating material in medicine to achieve a controlled release drug delivery due to its hydrogel-forming properties [10]. The gelling properties of its guluronic residues with divalent and trivalent ions, such as $\mathrm{Fecl}_{+3}, \mathrm{Cacl}_{+2}, \mathrm{CaCO}_{3}, \mathrm{Alcl}_{+3}$, and $\mathrm{Bacl}_{+2}$ etc. permit the formation of alginate matrices for gels, beads, and pellets [11].

Alginates have mucoadhesive property, but the cross-linked alginates are usually fragile in nature $[12,13]$. Therefore, to formulate various crosslinked alginate mucoadhesive microcapsules for controlled drug delivery. The effect of polymer blending or coating on the preparation of alginate microspheres containing ezetimibe [14]. Alginate is known to be nontoxic when taken orally and also has a protective effect on the mucous membranes of the upper gastrointestinal tract [15]. The dried alginate beads have the property of reswelling and thus they can act as a controlled release system [16] and properties of the beads prepared by ionotropic gelation are influenced by formulation, selection of method and other processing parameters.

This statement is supported by several papers reporting that drug release and/or encapsulation efficiency is dependent on type of drug and its characteristics [17], type and concentration of polymer inclusion of various additives [18-23].

Chemically it is a polysaccharide composed of varying proportions of D-mannuronic acid and L-guluronic acid residues which are arranged in sequences of MM or GG blocks interspersed with MG blocks. The current uses of alginate-based devices are mainly used to encapsulation of various classes of therapeutic agents.

Ezetimibe [24] is classified as a class II drug based on the Biopharmaceutical Classification System because of its low water 
solubility and high permeability. Despite the high permeability of class II drugs [25], they often show low oral bioavailability because of their slow and limited drug release in the gastrointestinal fluid. It acts as an anti-hyper lipedemic drug and shows its action by inhibiting intestinal cholesterol absorption [26].

Ezetimibe shows $35 \%$ bioavailability due to poor solubility. Ezetimibe was entrapped in aluminium alginate beads prepared with sodium alginate by choosing the ionotropic gelation (syringe method) using aluminium chloride as a curing agent.

The drug was incorporated either into preformed aluminium alginate gel beads or along with polymer (sequential method) or incorporated simultaneously during the gelation stage (simultaneous method). The beads were subjected for particle size and surface morphology using optical microscopy and SEM studies. Alginate beads produced by the sequential method had gained higher drug entrapment relatively.

Drug entrapment in the sequential method was higher with increased in curing agent and polymer concentration but lower with increased drug concentration. In the simultaneous method, drug entrapment was higher when polymer and drug concentration were increased and also rose to a certain extent with increase in $\mathrm{Alcl}_{+3}$ concentration, where further increase resulted in lower drug loading. FTIR studies revealed that there is no interaction between drug and other excipients. Release characteristics of the ezetimibe cross-linked aluminium alginate beads were studied separately in both simulated gastric fluid for 2 $\mathrm{h}$ and simulated intestinal fluid for $9 \mathrm{~h}$.

In this study, microparticles/beads were prepared by ionic gelation technique [27]. Ezetimibe was incorporated into aluminium alginate beads by individual methods naming sequential and simultaneous methods. The effect of polymer and $\mathrm{Alcl}_{+3}$ concentrations and that of ezetimibe concentration on drug entrapment (drug loading), the effect of the method (in adding drug) and drug release characteristics were studied.

\section{MATERIALS AND METHODS}

Ezetimibe (Aizant, Hyderabad, India), sodium alginate $45 \mathrm{cps}(1 \%$ w/v solution in water at $25^{\circ} \mathrm{C}$ ), carbopol 934P (SD Fine Chemicals. Mumbai), aluminium chloride dihydrate, potassium dihydrogen phosphate, potassium hydroxide pellets (E. Merck, India), sodium lauryl sulphate were used. All other used reagents were of analytical grade.

\section{Optimisation of curing agent}

In current study sodium, alginate beads were prepared by using divalent and trivalent curing agent at $5 \% \mathrm{w} / \mathrm{v}$ level and curing has done for a period of $60 \mathrm{~min}$. to get soft beads to harder in/along the surface of formed alginate beads during the curing period. Different curing agents like sodium sulphate, calcium carbonate and barium chloride beads of $2 \%, 3 \%$ were used, of all best was found to be aluminium chloride at $5 \%$ $\mathrm{w} / \mathrm{v}$ level and same implemented for further studies as a hardening agent in the preparation of hydrogel beads.

\section{Preparation of alginate hydrogel beads}

Cross-linked alginate beads were prepared by adopting ionic gelation technique which is said to be easiest and simple method of all microencapsulation techniques. For bead formation, $100 \mathrm{ml}$ of a 2.5 and $3 \% \mathrm{w} / \mathrm{v}$ aqueous solution of sodium alginate was discharged dropwise from a syringe with a size- 22 needle into $100 \mathrm{ml}$ of an aqueous $5 \% \mathrm{w} / \mathrm{v}$ Aluminium chloride solution being stirred at $200 \mathrm{rpm}$. Keep distance between needle to the curing medium was $15 \mathrm{cms}$. in gap to get stable beads. Since alginate gel can easily be formed by this ionic interaction in aqueous medium [28] gel beads are commonly obtained by dropping solutions of sod. Alginate into a solution of electrolyte.

The concentration of $\mathrm{Alcl}_{+3}$ in the solution ranged from $1 \% \mathrm{w} / \mathrm{v}$ to $3 \%$ $\mathrm{w} / \mathrm{v}$. The stirring was continued for one hour and make sure that no air bubble should be appear throughout polymer solution and subject to sonicator for more than one hour and formed the aluminium alginate beads were separated by filtration, obtained beads washed with distilled water three times continuously to remove traces of curing medium, and make air dried overnight $[29,30]$.

Table 1: Composition of ezetimibe-loaded aluminium alginate beads

\begin{tabular}{|c|c|c|c|}
\hline Batch code & Sodium alginate mixture* (gms.) & Aluminium chloride concentration $(\% \mathrm{w} / \mathrm{v})$ & Ezetimibe $(\% \mathrm{w} / \mathrm{v})$ \\
\hline A1 & 2.5 & a & 1 \\
\hline A2 & 2.5 & 3 & 2 \\
\hline A3 & 3 & 3 & 1 \\
\hline A4 & 3 & 3 & 2 \\
\hline B1 & 2.5 & 4 & 1 \\
\hline B2 & 2.5 & 4 & 2 \\
\hline B3 & 3 & 4 & 1 \\
\hline B4 & 3 & 4 & 2 \\
\hline C1 & 2.5 & 5 & 1 \\
\hline $\mathrm{C} 2$ & 2.5 & 5 & 2 \\
\hline C3 & 3 & 5 & 1 \\
\hline $\mathrm{C} 4$ & 3 & 5 & 2 \\
\hline
\end{tabular}

*Each Sodium alginate mixture contains 0.3 gms of carbopol 934P

Drug loading was carried out by two methods, naming as the sequential method and the simultaneous method. In the first method, aluminium alginate beads were prepared as described in the previous paragraph.

The wet beads were then immersed in curing solution and stirred for $1 \mathrm{hr}$ in a solution containing ezetimibe (concentration ranging from 2-3 \% w/v), filtered and washed with distilled water. Ezetimibe loaded calcium alginate beads were obtained by drying at room temperature.

In the simultaneous method, the gelation of beads by aluminium ions occurred simultaneously with the drug loading into the beads. The sodium alginate solution was introduced dropwise into $\mathrm{Alcl}_{+3}$ solutions (concentration ranging from $2-4 \% \mathrm{w} / \mathrm{v}$ ) which also contained ezetimibe (concentration ranging from $2-3 \% \mathrm{w} / \mathrm{v}$ ). After
$1 \mathrm{~h}$. of curing, the beads were removed from the counterion solution i.e. Alcl +3 .

\section{Determination of solubility}

The solubility of ezetimibe in various medium like water, acetate buffer, phosphate buffer pH 6.8 and in few cases SLS was added in small concentrations to affect solubility (as per standard monograph) were studied by preparing saturated solutions of the drug in these solvents.

Saturated solutions were prepared by adding an excess amount of drug to the vehicle in a screw capped vials, were kept on an orbital shaker for $24 \mathrm{~h}$. at $25{ }^{\circ} \mathrm{C}$. The screw-capped vials were centrifuged at $500 \mathrm{rpm}$ for further solubility of crystalline material and thereby obtaining a clear supernatant. 
After $1 \mathrm{hr}$. centrifugation, accurately measured quantities of the filtered supernatant solutions were further diluted with methanol and analysed spectrophotometrically at $232 \mathrm{~nm}$ for their drug content determinations.

\section{Determination of drug content}

For determination of drug content, $10 \mathrm{mg}$ of beads were placed in $100 \mathrm{ml}$ of 6.8 phosphate buffer for $12 \mathrm{~h}$. The filtered solution was measured for ezetimibe content using a UV spectrophotometer at $232 \mathrm{~nm}$. Drug content was computed using a calibration curve $\left(\mathrm{R}^{2}=\right.$ 0.9998 ) prepared using solutions with concentrations of $1-6 \mu \mathrm{g} / \mathrm{ml}$ of ezetimibe.

The drug loading capacity of the beads was then computed according to the following equation.

$$
\text { Drug loading }(\%)=\frac{\text { Total amount of drug in particles }}{\text { Weight of the particles }} \times 100
$$

\section{FTIR analysis}

The FTIR analysis of pure drug, polymer, and drug loaded beads prepared by both the methods were analysed with FTIR spectrophotometer (Shimadzu FTIR-8400, Japan). All the samples were crushed with potassium bromide to get pellets at $600 \mathrm{~kg} \mathrm{~cm}^{-2}$. Spectral scanning was done in the range of $400-4000 \mathrm{~cm}^{-1}$. FTIR spectra of drug and polymer blend which indicated that no major interaction occurred between the functional groups of the ezetimibe and used polymer blend upon mixing [31].

\section{Scanning electron microscopy}

Scanning electron microscopic (Joel-LV-5600, USA, with a magnification of $250 \mathrm{x}$ ) photographs were obtained to give information about identifying and confirm solid dispersions crystals nature and morphological characters of the crystals.

\section{X-Ray diffraction}

For physical state characterization of ezetimibe, $\mathrm{x}$-ray diffraction studies were performed for pure drug and solid dispersion. XRD study was carried out with (Bruker D8 advance Bruker, Germany). Measurements were performed at a voltage of $45 \mathrm{kV}$ and $40 \mathrm{mv}$. The diffraction angle $2 \theta$ was set from 50 to 490 , and the scanned rate was $2 \mathrm{~min}$.

\section{Determination of swelling properties}

The dynamic swelling property of microcapsules in the both i.e. acidic and $6.8 \mathrm{pH}$ phosphate buffer medium was determined. Microcapsules of known weight were placed in dissolution solution for specified medium and the swollen microcapsules were collected by decantation and the wet weight of the swollen microcapsules was determined by first blotting the particles with filter paper to remove absorbed water on the surface and then weighing immediately again on an electronic balance. The difference in given values give the percentage of swelling of microcapsules in the dissolution media and it was calculated by using equation.

\section{$\mathrm{S}_{\mathrm{w}}=\left(\mathrm{W}_{\mathrm{t}}-\mathrm{W}_{\mathrm{o}}\right) / \mathrm{W}_{\mathrm{o}} \mathrm{X} 100$}

Where $S_{w}=$ percentage of swelling of microcapsules/beads, $W_{t}=$ weight of the microcapsules at time $t$, and $\mathrm{W}_{0}=$ initial weight of the microcapsules.

\section{Erosion studies}

The dried alginate beads were weighed and placed in a dissolution basket, followed by dissolution in phosphate buffer $(\mathrm{pH} \mathrm{6.8)} \mathrm{at} 70$ rpm. After every $1 \mathrm{hr}$. interval, the beads were removed from the basket and oven-dried at $40{ }^{\circ} \mathrm{C}$. The water was removed, and the beads were reweighed. The beads were then placed back in the basket. This process lasted $11 \mathrm{~h}$. Erosion study was performed using the following formula.

$$
\% \text { weight loss }=\left(w_{0}-w_{1}\right) \times 100 / w_{0}
$$

Where $\mathrm{W}_{\mathrm{o}}=$ Initial beads, weight and $\mathrm{W}_{1}=$ weight of beads after drying.

\section{Drug release studies}

Drug release studies were carried out in triplicate using a USP II tablet dissolution test apparatus (Model-TDP-06P, Electro Lab, Mumbai, India) at $37 \pm 0.5^{\circ} \mathrm{C}$ and at $70 \mathrm{rpm}$ for $11 \mathrm{~h}$. The beads of each formulation were placed in simulated gastric fluid $(900 \mathrm{ml}$, $0.1 \mathrm{~N} \mathrm{Hcl}, \mathrm{pH} 1.2$ ) for the first $2 \mathrm{~h}$. and then in simulated intestinal fluid ( $900 \mathrm{ml}$, Phosphate buffer solution, $\mathrm{pH}$ 6.8) up to total $11 \mathrm{~h}$. A 5 $\mathrm{ml}$ aliquot of the dissolution fluid was withdrawn at different regular intervals of time and replaced immediately with the same $5 \mathrm{ml}$ volume of fresh media to maintain sink conditions. The aliquots, following suitable dilution, were analysed for drug content by using a spectrophotometer at a wavelength of $232 \mathrm{~nm}$.

\section{RESULTS AND DISCUSSION}

\section{Solubility data}

The solubility of ezetimibe was tested in water, acetate buffer, and pH 6.8 phosphate buffer was tested and in few cases, sodium lauryl sulphate was added to effect solubility of ezetimibe. Samples were analysed for drug solubility after suitable dilutions and reading were taken by using a UV-Visible spectrophotometer at $232 \mathrm{~nm}$. The solubility results were predicted in following table 2 .

Table 2: Solubility values with various buffers

\begin{tabular}{lll}
\hline S. No. & Medium & Solubility mg/ml $^{\mathbf{a}}$ \\
\hline 1 & $0.1 \mathrm{NHcl}$ & $0.0090 \pm 3.32$ \\
2 & Acetate buffer pH 3 & $0.0000 \pm 2.31$ \\
3 & $0.05 \mathrm{M}$ Acetate buffer pH 3 & $0.0000 \pm 1.56$ \\
4 & $0.3 \%$ SLS in 0.05M Acetate buffer pH 3 & $0.0291 \pm 4.67$ \\
5 & $0.5 \%$ SLS in 0.05M Acetate buffer pH 3 & $0.0412 \pm 3.67$ \\
6 & Phosphate buffer 6.8 & $0.0311 \pm 7.67$ \\
7 & Phosphate buffer 6.8+0.5 \% SLS & $0.0451 \pm 8.91$ \\
\hline
\end{tabular}

${ }^{\mathrm{a}} n=3$ (average $\pm \mathrm{SD}$ )

\section{Particle size}

Determination of particle size was done by using optical microscope method. Particle size was in the range of $75-80 \mathrm{~mm}$ in diameter for the beads obtained using the sequential method, and $90-100 \mathrm{~mm}$ to the beads for those from the simultaneous method. The particle size of the beads prepared by the simultaneous method decreased with higher $\mathrm{Alcl}_{+3}$ concentrations. Size of the beads was observed more in $\mathrm{A} 2, \mathrm{~A} 4, \mathrm{~B} 2, \mathrm{~B} 4, \mathrm{C} 2$ and $\mathrm{C} 4$ batches reason was owing to the presence of higher drug concentration. Based on high levels of sodium alginate in batches, A3, A4, B3, B4, C3 and C4 attained little bit higher in diameter of beads than rest of batches.

\section{FTIR data}

FTIR studies have carried out and the report has confirmed that there will be no reaction between drug and excipients and compatibility atmospheres among the all used excipients. Spectral variations originate due to alteration in bonds that exhibit 
characteristic vibrational frequencies, leading to frequency shifts and splitting in absorption peaks. Spectrum of pure ezetimibe displayed characteristic peaks from 3300 to $3400 \mathrm{~cm}-1$ (intermolecular hydrogen bonded, $\mathrm{O} \mathrm{H}$ stretch), from 1400 to $1600 \mathrm{~cm}^{-1}$ (aromatic C C stretch), $1221 \mathrm{~cm}^{-1}$ (C F stretch), $834 \mathrm{~cm}^{-1}$ (ring vibration due to para-substituted benzene) and $2916 \mathrm{~cm}$ -
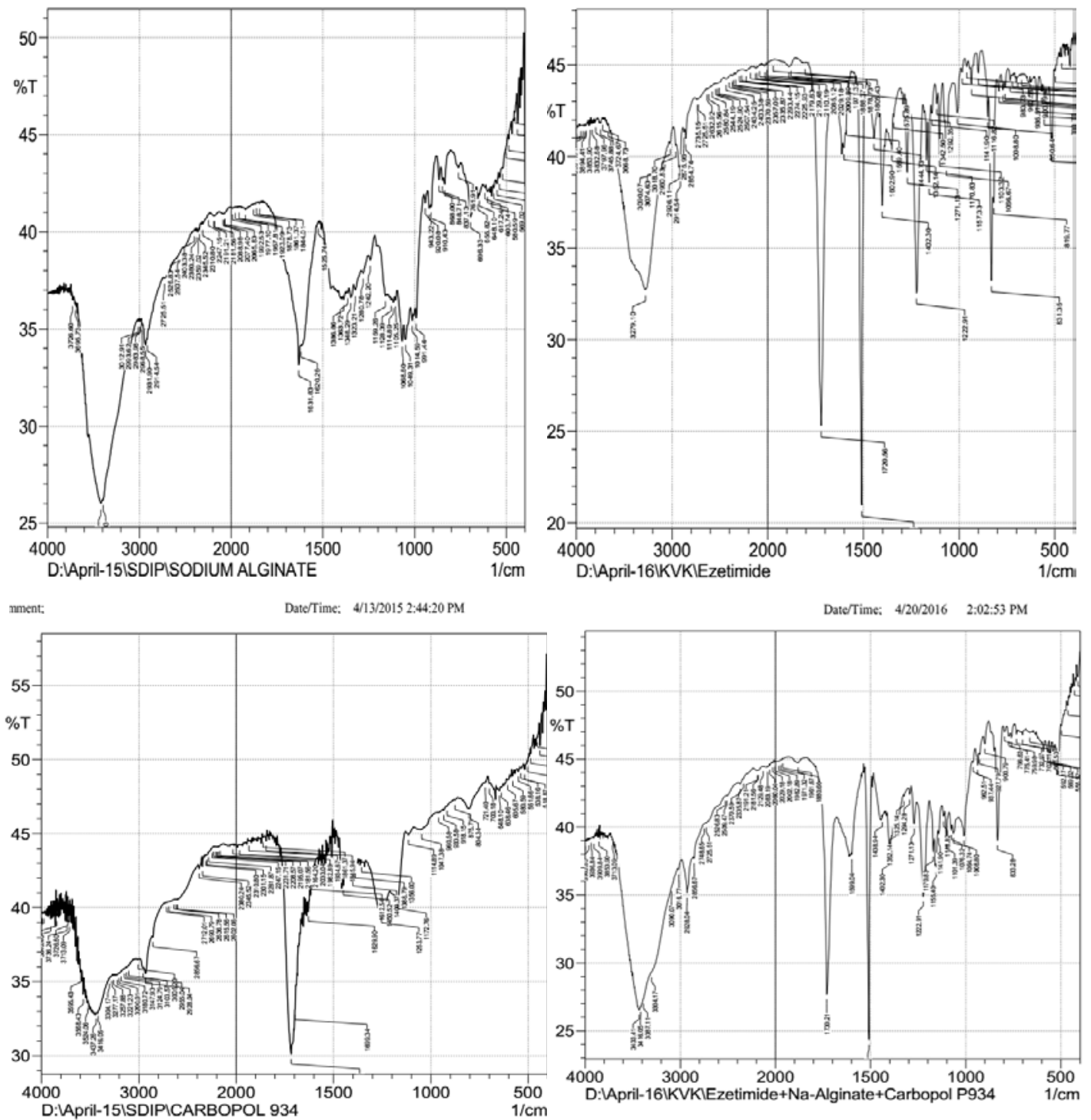

Date/Time: 4/202016 2:31:14 PM
${ }^{1}$ (sp3 CH stretch) [32.33]. FTIR spectra of the physical mixture also showed peaks at similar position. So it can be concluded that there was no occurrence of possible interaction between drug and used hydrophilic carrier sodium alginate and carbopol 934P. the FTIR spectra of the different compound and physical mixture were illustrated in above fig. 1 .

Fig. 1: FTIR Spectras of pure ezetimibe, pure sodium alginate, pure carbopol 934P and physical mixture

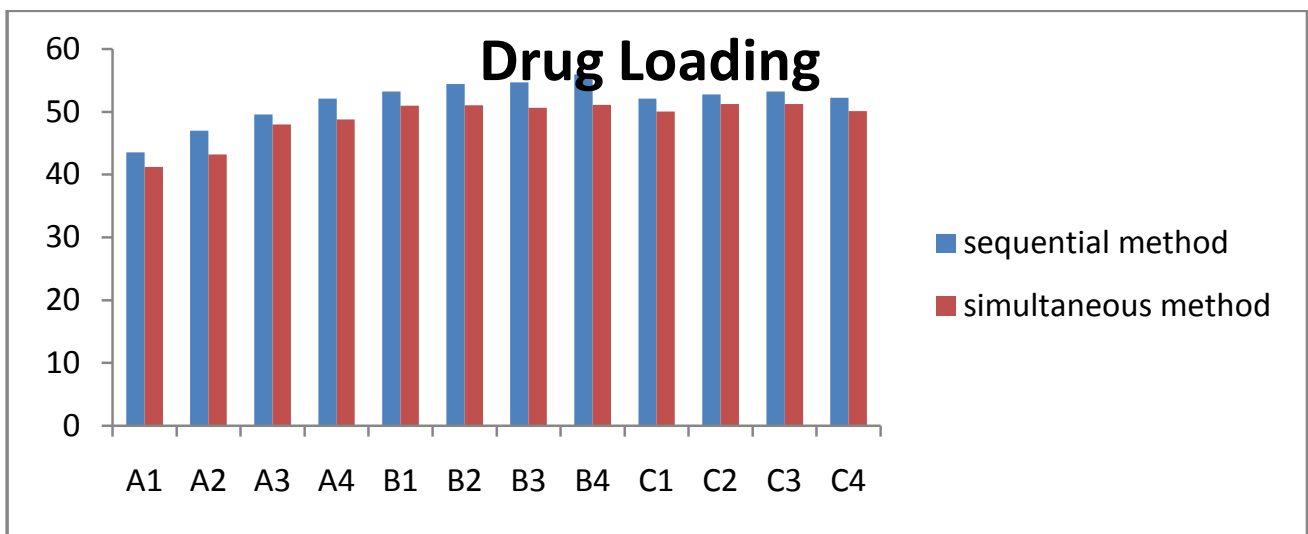

Fig. 2: Drug loading of various batches in sequential and simultaneous methods, Data are $(\mathrm{n}=3$ ) (average \pm Standard deviation $(\mathrm{SD})$ 


\section{Scanning electron microscopy}

The surface of the alginate microspheres was rough due to a higher concentration of drug and sodium alginate.
The addition of alginate in higher concentration by ionic gelation technique it forms a rough surface which was confirmed by SEM studies.

The SEM photographs are depicted in fig. 3.

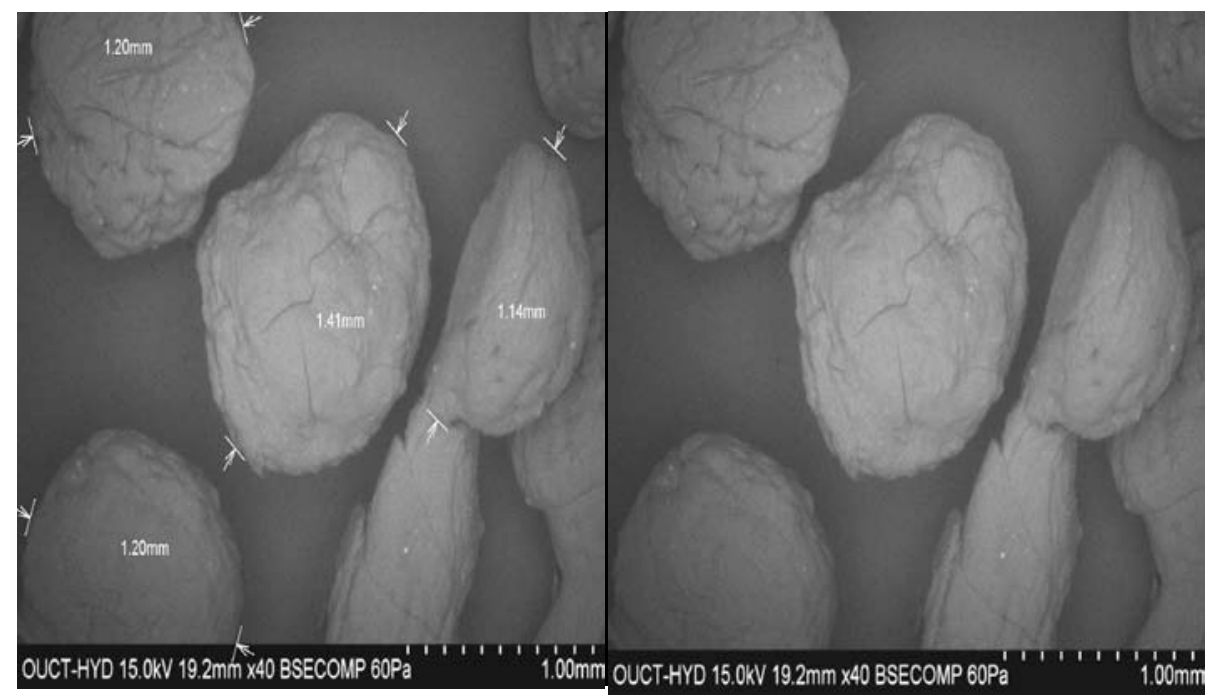

Fig. 3: SEM picture of dried sodium alginate beads

In C4 formulation beads were appeared more in diameter of beads and smoothness than compared to the C3 formulation the reason is contributed to the high concentration of drug substance. The SEM photographs of the drug-loaded beads show that the drug is distributed equally in the polymeric
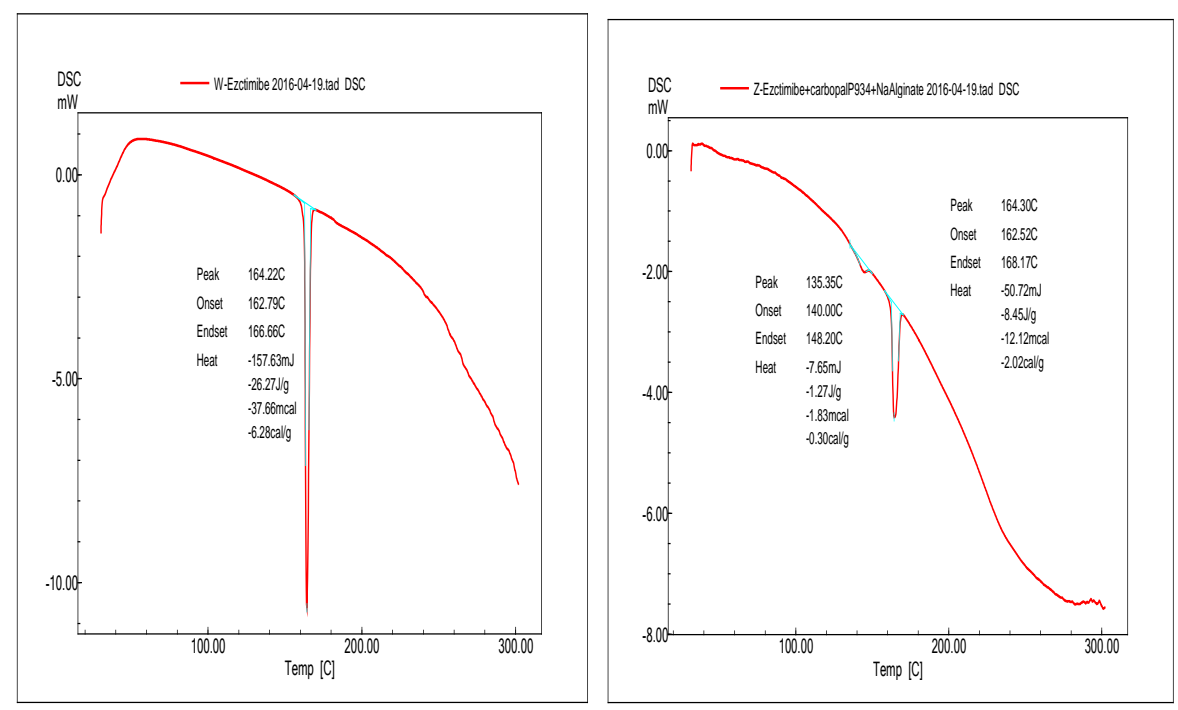

Fig. 4: DSC spectras of pure ezetimibe (left) and ezetimibe with carbopol 934P mixture (right)

\section{Drug loading}

Drug loading was found to be in the range of $43-56 \%$ for beads prepared by the sequential method and $41-49 \%$ for the simultaneous method. Drug loading was found to be directly proportional to polymer concentration in both the methods. The effect of crosslinking agent and drug concentration on drug loading, however, it was different in both cases. Higher loading efficiency was obtained as the concentration of alginate increased. This may be attributed to the greater availability of no. of active aluminium binding sites in the polymeric chains and increasing the amount of matrix without having any coat, which further confirms that this system is a polymeric matrix system for beads prepared by both sequential and simultaneous methods. The surface of beads after dissolution was softer (swelled nature) than before dissolution. 
For the simultaneous method, batch B4 had the highest drug loading (58\%). When the drug loading was carried out by the simultaneous method with the gelation of alginate by $\mathrm{Al}_{+3}$, the counterion solution i.e. curing medium contained the drug molecule and $\mathrm{Alcl}_{+3}$. The drug molecules and $\mathrm{Al}_{+3}$ ions migrate/diffuse in the same pathway, from the solution into the sod. Alginate solution, and competed simultaneously for common binding sites in the chains of alginate polymer. The amount of associated cross-linked $\mathrm{Al}_{+3}$ ions (and the API drug) in the beads was a function of the concentration ratio of $\mathrm{Alcl}_{+3}$ : ezetimibe in the solution.

The increase in $\mathrm{Alcl}_{+3}$ as a curing agent concentration from 3 to $5 \%$ $\mathrm{w} / \mathrm{v}$ and polymer concentration from 2.5 to $3 \% \mathrm{w} / \mathrm{v}$ resulted in an increase in the drug entrapment. Drug entrapment is decreased from $62 \%$ to $42 \%$ when the drug concentration was increased from $2-3 \%$ for the same.

Similarly, in the simultaneous method increase in drug entrapment from $47 \%$ to $58 \%$, from $52 \%$ to $58 \%$ and from $55 \%$ to $58 \%$ was observed for corresponding changes in the $\mathrm{Alcl}_{+3}$, polymer and drug concentration.

\section{Swelling study}

It was cleared that by the increasing of alginate concentration, the swelling index increase this was due to the high absorption capacity of alginate. The presence of hydrophobic carbopol 934P in the beads maintained swelling for a longer time than Na-alginate alone, because hydrophobic carbopol 934P provided higher viscosity levels than Na-alginate, thus preventing a quick loss of the initial bead shape. All microbeads began to swells presumably due to an increase in the intensity of electrostatic repulsive forces at a $\mathrm{pH}$ above the pKa of the uronic acid groups on the alginate.

Also swelling of the dry microspheres can be attributed to the hydration of the hydrophilic groups of alginate and carbopol 934P. In this case free water penetrates inside the microspheres in order to occupy the pores among polymer chains, contributing to a greater swelling degree. The rate of swelling was in following the order of decreasing:

\section{$5 \% \mathrm{w} / \mathrm{v} \mathrm{Alcl}_{3}<4 \% \mathrm{w} / \mathrm{v} \mathrm{Alcl}_{3}<3 \% \mathrm{w} / \mathrm{v} \mathrm{Alcl}_{3}$}

As the percentage of curing agent's increases, the rate of swelling becomes lowers, the reason was contributed to the gaining of the tight and compact arrangement of alginate beads and becomes a shrink in its orientation like.

\section{Erosion studies}

Results are plotted in fig. In erosion tests run in phosphate buffer (pH 6.8), both types of beads exhibited an initial increase in volume. Na-alginate beads started to erode significantly at 30-45 min, losing their spherical shape after 130-160 min, whereas the cross-linked formulations kept their shape throughout the test $50 \%$ integrity after $4 \mathrm{~h}$ ). This feature was also observed throughout the drug release tests and is probably the main cause underlying the prolonged release of the drug from the beads.

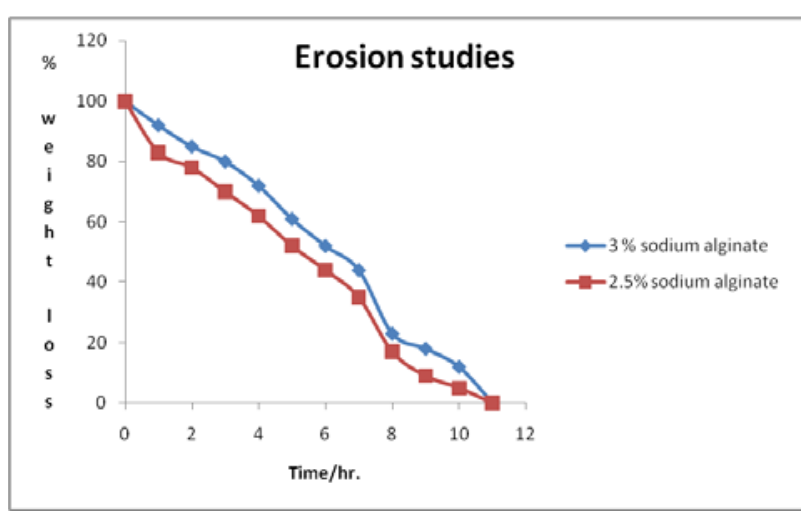

Fig. 5: Erosion profile of $3 \%$ and $2.5 \% \mathrm{w} / \mathrm{v}$ of sodium alginate (each contains $0.3 \% \mathrm{w} / \mathrm{v}$ of carbopol 934P). $n=3$ (average \pm SD)

\section{In vitro studies}

The dissolution studies carried by using two different mediums naming acidic medium and 6.8 phosphate buffer medium for a period of 2 and $9 \mathrm{~h}$. at room temperature under $75 \mathrm{rpm}$ by using USP dissolution apparatus method. In first $2 \mathrm{hr}$. swelling of beads is to a minimum extent in the presence of acidic medium, which indicated that there was a slow and controlled release of drug for all the formulation but in 6.8 phosphate buffer medium the rate of swelling is tripled than acidic medium. From above data, it was concluded that the amount of release of drug with respect to time is a function of $\mathrm{pH}$ basis. Each readings were taken as the average of three.

In the case of water soluble polymers, dissolution of the polymer results in a gradual erosion of this gel layer.

However, at higher concentrations, the polymer chains entangle to a greater degree culminating in "virtual cross-linking" and therefore the formation of a stronger medium compact gel layer. The release studies were conducted in triplicate.

In Simulated gastric fluids, alginate beads prepared by the sequential method, ezetimibe releases about less than $21 \%$ drug in the first $2 \mathrm{~h}$. The rest of the drug then releases in the SIF ie. 6.8 phosphate buffer continuing for next $9 \mathrm{~h}$. whereby it releases remaining almost $82-89 \%$ of the drug.

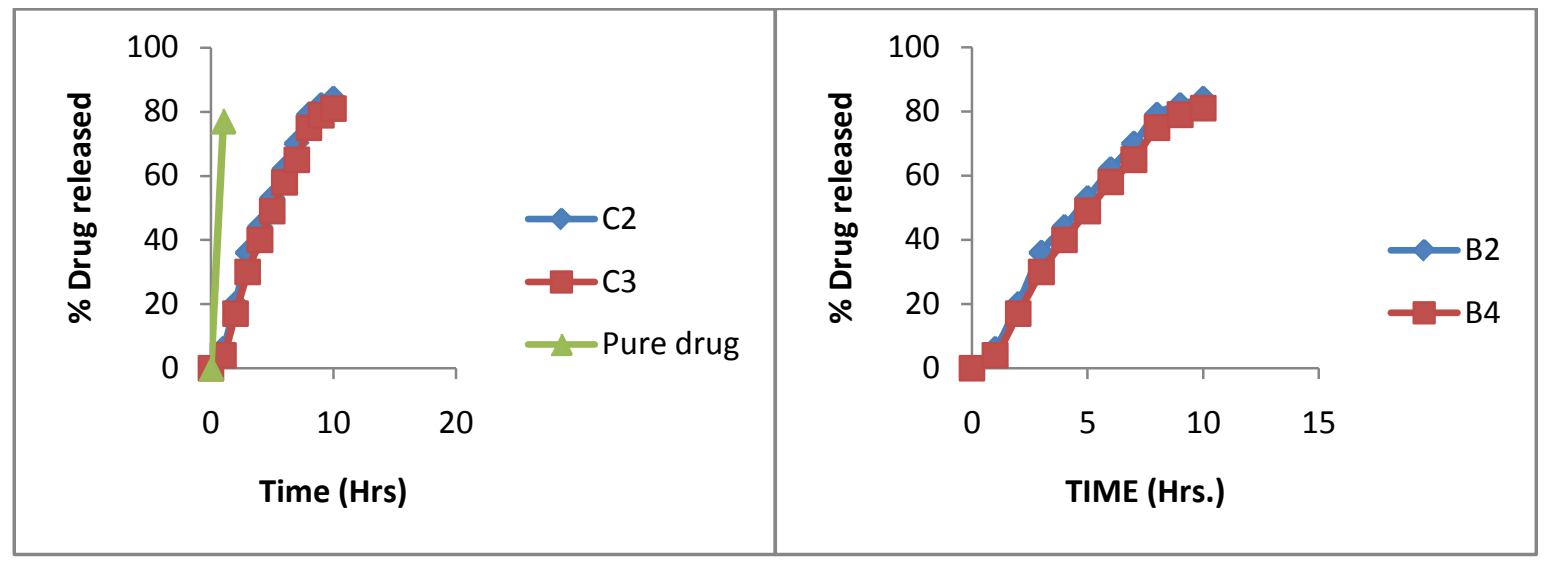

Fig. 6: Effect of sodium alginate concentration on ezetimibe release from aluminium alginate beads (right fig.) Sequential method (left fig.) Simultaneous method. $n=3$ (average \pm SD) 


\section{Effect of polymer}

To study the effect of sodium alginate concentration on drug release, two different concentrations, 2.5 and $3 \% \mathrm{w} / \mathrm{v}$ were used. Fig. 6 show the influence of the concentration of sodium alginate polymer solution on ezetimibe release behaviour from aluminium alginateloaded beads prepared by the sequential and simultaneous methods.

Lower concentrations of sodium alginate contribute to faster drug release, with $87.05 \pm 0.778 \%$ of drug release within $11 \mathrm{~h}$. using the sequential method. The increase in alginate concentration gives higher viscosity which makes slow the release pattern of drug particles. Thus, the results indicate that drug release was directly proportional to the addition of sodium alginate concentration. The cross-linking of sodium alginate with $\mathrm{Alcl}_{+3}$ is based on the tight junction formed between the guluronic acid residues. The number of the apparent cross-linking points formed within increases with increasing alginate concentration $(2.5-3 \% \mathrm{w} / \mathrm{v})$ in the formulation.

This can be correlated with the particle size studies where, as sodium alginate concentration increased, particle size get decreased, but at higher $\left(\mathrm{Alcl}_{3} 5 \% \mathrm{w} / \mathrm{v}\right)$ the reason due to the formation of a rigid and compact matrix, consequently retarding/slower ezetimibe release. The regression coefficient values and slope values of formulations were expressed in table no.3.
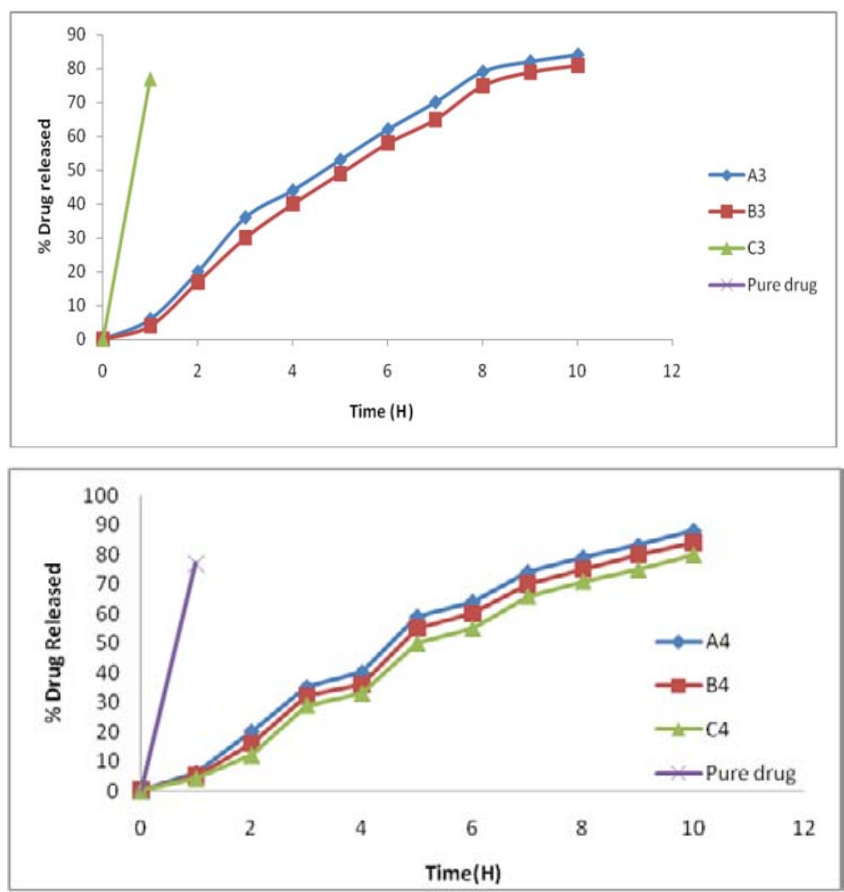

Fig. 7: Effect of $\mathrm{Alcl}_{+3}$ concentration on ezetimibe release from cross-linked aluminium hydrogel alginate beads (above graph) Sequential method (below graph) Simultaneous method, $n=3$ (average \pm SD)

\section{Effect of curing agent}

The release behaviour of aluminium alginate beads prepared by the sequential method with different concentrations of Alcl +3 (3, 4 and 5 $\% \mathrm{w} / \mathrm{v}$ ) is indicated in fig. 7. Drug release was sustained from $22 \%$ to $29 \%$ in SGF for $2 \mathrm{~h}$, and $78 \%$ to $80 \%$ in SIF fluid up to $9 \mathrm{~h}$. With increasing in $\mathrm{Alcl}_{+3}$ concentration. Thus, in the sequential method, as the concentration of crosslinking agent increased, drug release decreased because of a tight compact network of alginate beads which was gained of raising levels of the curing agent. This may be explained by the increase in the gel strength as the aluminium ions increased.

Beads prepared with $5 \% \mathrm{w} / \mathrm{v} \mathrm{Alcl}_{+3}$ showed the most sustained release effect due to more crosslinking resulting in the formation of a more rigid gel network and an increase in no. of binding sites leads to the greater sustained release of drug and more drug entrapment than $3 \% \mathrm{Alcl}_{+3}$. It was also found that further increase in the concentration of curing agent (up to $5 \% \mathrm{w} / \mathrm{v}$ ) did not enhance the drug loading, this could be due to attainment of possible saturation of aluminium binding sites in the guluronic acid chain, preventing further aluminium ion entrapment.
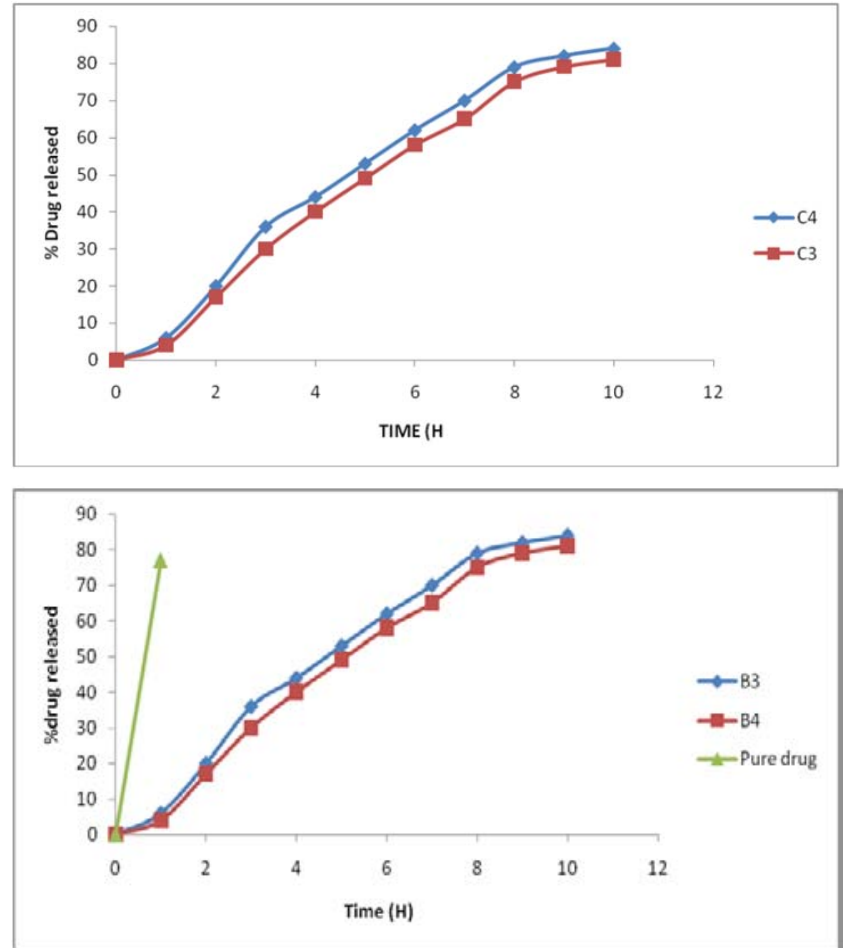

Fig: 8. Effect of ezetimibe concentration on ezetimibe release from the aluminium alginate beads (above) Sequential method (below) Simultaneous method. $n=3$ (average \pm SD)

In the case of ezetimibe loaded aluminium alginate beads prepared by the simultaneous method, similar behavior was also observed.

It was confirmed by analysis of particle size studies, where the size of the beads was found to be smaller as the concentration of crosslinking agent increased [34-38] i.e. Beads made with 5\% $\mathrm{w} / \mathrm{v} \mathrm{Alcl}_{+3}$ yields small size beads compared to 3 and $4 \% \mathrm{w} / \mathrm{v}$ $\mathrm{Alcl}_{+3}$. It was also evident from the literature that diffusion of the drug from alginate matrix decreased as the concentration of $\mathrm{Alcl}_{+3}$ solutions increased in the sequential method, probably due to more no. of cross-linking sites with sodium alginate polymer. In the simultaneous method, ezetimibe release was sustained from $24 \%$ to $30 \%$ in SGF for $2 \mathrm{~h}$, and from $89 \%$ to $90 \%$ in SIF up to $9 \mathrm{~h}$. But in the case of the simultaneous method, $3 \%$ crosslinking agent did not show much the highest sustained effect. This may be the reason of low drug loading.

Maximum swelling of beads was noted at 5-9 h. in the 6.8 phosphate buffer medium after which beads will experience both erosion and breakdown process take place resulting in decline in the index [39] behaviour results from slow erosion of cross-linked microbeads due to a slight degradation of alginate backbone into smaller fragments [40].

\section{Effect of drug concentration}

The effect of drug concentration during in vitro drug release study does not affect the drug release from the obtained aluminium alginate beads. In current research, the drug concentration ranged from 1 to $2 \% \mathrm{w} / \mathrm{v}$. only. To study the effect of the drug on release pattern shown in fig. no.10 ezetimibe release from beads prepared by the sequential method (batch C3), which contained 1\% w/v ezetimibe, shows a more sustained effect with a drug loading of about 53\%. 
However, in the case of the simultaneous method (batch B4), which contains $2 \% \mathrm{w} / \mathrm{v}$ drug; a more sustained effect with a drug loading of about $54-56 \%$ was evident. In the simultaneous method, less than $50 \%$ of drug loading occurs. The amount of drug release was highly dependent on drug loading.

The alginate disintegration was monitored by the exchange of $\mathrm{Al}_{+3}$ with potassium ions in the dissolution medium. The first phase might be for the negligible (i.e. less amount of drug release)dissociation of alginate beads in 6.8 phosphate buffer and the drug release mainly based on the principle of drug diffusion through the small pores and cracks on/along the surface of the ezetimibe beads. The second phase exhibited a burst-like release pattern, which was accompanied by alginate disintegration of beads in 6.8 phosphate buffer medium.
During dissolution process aluminium ions exchanges with 6.8 phosphate buffer causes the slow erosion of the beads, which increases the rate of drug release moderately.

The complete dehydration of alginate microspheres may develop a small degree of surface cracking which can facilitate the surface erosion of the beads upon rehydration. Erosion could occur through degradation of alginate backbone into smaller molecular weight components. The dried alginate beads swelled slightly in $0.1 \mathrm{~N} \mathrm{Hcl}$. for first $2 \mathrm{~h}$. At $\mathrm{pH} 6.8$ phosphate buffer the release was due to both diffusion and swelling and erosion principle mechanisms, which resulted in disaggregation/break down of alginate matrix structure leading to matrix erosion and dissolution of swollen ezetimibe microcapsules $[41,42]$.

Table 3: Release kinetics of all formulations

\begin{tabular}{|c|c|c|c|c|c|}
\hline Batch & Zero order & First order & Higuchi matrix* & Korsmeyer peppers* & Hixon crowell equation* \\
\hline A2 & $\mathrm{R}^{2}=0.830$ & $\mathrm{R}^{2}=0.914$ & $\mathrm{R}^{2}=0.910$ & $\begin{array}{l}\mathrm{R}^{2}=0.978 \\
\text { slope }=1.625 \mathrm{x}+0.507\end{array}$ & $\begin{array}{l}\mathrm{R}^{2}=0.921 \\
\text { slope }=0.863 \mathrm{x}+4.562\end{array}$ \\
\hline A3 & $\mathrm{R}^{2}=0.939$ & $\mathrm{R}^{2}=0.894$ & $\mathrm{R}^{2}=0.937$ & $\begin{array}{l}\mathrm{R}^{2}=0.798 \\
\text { slope }=1.427 \mathrm{x}+0.507\end{array}$ & $\begin{array}{l}\mathrm{R}^{2}=0.831 \\
\text { slope }=0.829 x+5.752\end{array}$ \\
\hline A4 & $\mathrm{R}^{2}=0.966$ & $\mathrm{R}^{2}=0.982$ & $\mathrm{R}^{2}=0.895$ & $\begin{array}{l}\mathrm{R}^{2}=0.845 \\
\text { slope }=1.539 \mathrm{x}+0.576\end{array}$ & $\begin{array}{l}\mathrm{R}^{2}=0.870 \\
\text { slope }=0.854 \mathrm{x}+5.173\end{array}$ \\
\hline B2 & $\mathrm{R}^{2}=0.962$ & $\mathrm{R}^{2}=0.985$ & $\mathrm{R}^{2}=0.897$ & $\begin{array}{l}\mathrm{R}^{2}=0.844 \\
\text { slope }=1.514 \mathrm{x}+0.565\end{array}$ & $\begin{array}{l}\mathrm{R}^{2}=0.856 \\
\text { slope }=0.799 \mathrm{x}+5.297\end{array}$ \\
\hline B3 & $\mathrm{R}^{2}=0.960$ & $\mathrm{R}^{2}=0.999$ & $\mathrm{R}^{2}=0.906$ & $\begin{array}{l}\mathrm{R}^{2}=0.825 \\
\text { slope }=1.503 \mathrm{x}+0.609\end{array}$ & $\begin{array}{l}\mathrm{R}^{2}=0.862 \\
\text { slope }=0.840 \mathrm{x}+5.292\end{array}$ \\
\hline B4 & $\mathrm{R}^{2}=0.976$ & $\mathrm{R}^{2}=0.959$ & $\mathrm{R}^{2}=0.858$ & $\begin{array}{l}\mathrm{R}^{2}=0.901 \\
\text { slope }=1.616 \mathrm{x}+0.449\end{array}$ & $\begin{array}{l}\mathrm{R}^{2}=0.912 \\
\text { slope }=0.849 \mathrm{x}+4.685\end{array}$ \\
\hline $\mathrm{C} 2$ & $\mathrm{R}^{2}=0.965$ & $\mathrm{R}^{2}=0.985$ & $\mathrm{R}^{2}=0.906$ & $\begin{array}{l}\mathrm{R}^{2}=0.837 \\
\text { slope }=1.520 \mathrm{x}+0.582\end{array}$ & $\begin{array}{l}\mathrm{R}^{2}=0.862 \\
\text { slope }=0.831 \mathrm{x}+5.222\end{array}$ \\
\hline $\mathrm{C} 3$ & $\mathrm{R}^{2}=0.979$ & $\mathrm{R}^{2}=0.983$ & $\mathrm{R}^{2}=0.882$ & $\begin{array}{l}\mathrm{R}^{2}=0.880 \\
\text { slope }=1.596 \mathrm{x}+0.489\end{array}$ & $\begin{array}{l}\mathrm{R}^{2}=0.885 \\
\text { slope }=0.842 \mathrm{x}+4.894\end{array}$ \\
\hline $\mathrm{C} 4$ & $\mathrm{R}^{2}=0.975$ & $\mathrm{R}^{2}=0.979$ & $\mathrm{R}^{2}=0.837$ & $\begin{array}{l}\mathrm{R}^{2}=0.914 \\
\text { slope }=1.629 \mathrm{x}+0.429\end{array}$ & $\begin{array}{l}\mathrm{R}^{2}=0.916 \\
\text { slope }=0.863 \mathrm{x}+4.539\end{array}$ \\
\hline
\end{tabular}

${ }^{*} n=3$ (average $\pm \mathrm{SD}$ )

\section{CONCLUSION}

Ezetimibe was entrapped in aluminium alginate beads prepared with hydrophilic polymer sodium alginate and retarding material carbopol 934P by the using ionotropic gelation method. The method developed is easy, fast and reproducible. The addition of drug was incorporated by two methods, sequential and simultaneous methods. Beads produced by the former method had higher drug entrapment. The beads were evaluated for drug entrapment, particle size, erosion studies and in vivo studies in both simulated gastric and simulated intestinal fluid i. e 6.8 phosphate buffer for a period of 11 $\mathrm{h}$. The drug entrapment in the sequential method increased with increased $\mathrm{Alcl}_{+3}$ concentration and polymer concentration but decreased with higher drug concentration. But in the simultaneous method, drug entrapment was higher with polymer and drug concentration increase and rose to a certain extent with increase in the concentration of $\mathrm{Alcl}_{+3}$ and after the further increase, it decreased. From the analysis of results, While the increase in the concentration of sodium alginate obtained beads increased its sphericity, and mean diameter of the microspheres because of getting high viscosity on addition. The concept was cleared that drug release was dependent upon the quantity of polymer and it was confirmed that it is directly proportional to the polymer concentration and conc. of the curing agent. The increase in conc. of curing agent i.e. Alcl $_{+3}$ concentration retarded the drug release in the sequential method.

The ratio of drug to polymer has an effect on drug release from the microbeads. The drug concentration exhibits a drug loading dependent effect on the release behaviour in both the methods. Thus, all formulations delivered their active ingredient by coupled diffusion and erosion mechanism and it exhibits zero order release mechanism [43-46]. The release data of ezetimibe was processed to understand the linear relationship i.e. kinetic principles. The value of $\mathrm{n}$ is 1.492 for C3 batch; $\mathrm{n}$ value confirms hydrogel alginate beads that drug release from beads was followed super case-II transport mechanism control by swelling and relaxation of polymer chains.

On the basis of above-obtained data, it can be concluded that beads produced by the sequential method have achieved higher drug entrapment. Beads produced by simultaneous yields larger beads in diameter. The concept was cleared that drug release was dependent upon the quantity of polymer and increase in cons. of aluminium chloride retarded the drug release in the sequential method. Prepared cross-linked alginate beads enhance the dissolution of ezetimibe and the rate of oral bioavailability and also reduces the fluctuations in the oral bioavailability, also reduces the fluctuations in the oral bioavailability and improving the safety, and improve the efficacy of the active ingredient [47].

\section{ACKNOWLEDGMENTS}

The author thankful to Aizant labs, Hyderabad, India for providing pure drug sample and Osmania university, Hyderabad for taking FTIR spectras and SEM studies for finishing off a current research article and wish to coauthors for their valuable support to bring up to final stage of work and valuable technical support.

\section{CONFLICT OF INTERESTS}

Declared none

\section{REFERENCES}

1. Bhuvaneswari S, Manivannan S, Akshay M, Nify F. Formulation and evaluation of gastro retentive micro balloons of acebrophylline for the treatment of bronchial asthma. Asian J Pharm Clin Res 2016;5:105-111.

2. Mangond BS, Sreedhar V, Baraskar W, Kulkarni RV. Development and evaluation of gellan gum based hydrogel 
microbeads for controlled release of ketoprofen. Indian J Novel Drug Delivery 2009;1:32-5.

3. Rajinikanth PS, Sankar C, Mishra B. Sodium alginate microspheres of metoprolol tartrate for intranasal systemic delivery: development and evaluation. Drug Delivery 2003;10:21-8

4. McDowell RH. Properties of Alginates. $5^{\text {th }}$ Edition. Kelco International, London; 1986.

5. Bruneton J. Algae polysaccharides. In: Pharmacognosy, phyto chemistry medicinal plants. $2^{\text {nd }}$ ed. New York: Lavosier Publishing, Inc; 1999. p. 47-52.

6. Dhanaraju MD, Sundar VD, Nandha Kumar S, Bhaskar K. Development and evaluation of sustained delivery of diclofenac sodium from hydrophilic polymeric beads. J Young Pharm 2009;1:301.

7. Kikuchi A, Kawabuchi M, Sungihara M, Okano TS. Pulsed dextran releases from calcium alginate. J Controlled Release 1997; 47:21-9.

8. Iannuccelli V. Bead coating process via an excess of a crosslinking agent. Drug Dev Ind Pharm 1995;21:307-22.

9. Tomida H. Imipramine releases from Ca-alginate gel beads. Chem Pharma Bull 1993;41:1475-7.

10. Gray CJ, Dowsett J. Retention of insulin in alginate gel beads. Biotechnol Bioeng 1988;31:607-12.

11. Sarmento B, Martins S, Ribeiro A, Veiga F, Neufeld R, Ferreira D. Development and comparison of different nanoparticulate polyelectrolyte complexes as insulin carriers. Int J Pept Res Ther 2006;12:131-8.

12. Bajpai SK, Sharma S. Investigation of swelling/degradation behaviour of alginate beads crosslinked with $\mathrm{Ca} 2+$ and $\mathrm{Ba} 2+$ ions. React Funct Polym 2004;59:129-40.

13. Kroll E, Winnik FM, Ziolo RF. In situ preparation of nanocrystalline $\gamma-\mathrm{Fe}_{2} \mathrm{O}_{3}$ in iron (II) crosslinked alginate gels. Chem Mater 1996;8:1594-6.

14. Senuma Y, Lowe C, Zweifel Y, Hilborn JG, Marison I. Alginate hydrogel microspheres prepared by spinning disk automation. Biotechnol Bioeng 2000;67:616-22.

15. Kim CK, Lee EJ. The controlled release of blue dextran from alginate beads. Int J Pharm 1992;79:11-9.

16. Yotsuyanagi T, Ohkubo T, Ohhashi T, Ikeda K. Calcium-Induced gelation of alginic acid and $\mathrm{pH}$-sensitive reswelling of dried gels. Chem Pharm Bull 1987;35:1555-63.

17. Tang YD, Venkatraman SS, Boey YC, Wang LW. Sustained release of hydrophobic and hydrophilic drugs from a floating dosage form. Int J Pharm 2007;336:159-65.

18. El-Kamel AH, Al-Gohary OMN, Hosny EA. Alginate-diltiazem hydrochloride beads: optimisation of formulation factors, in vitro and in vivo bioavailability. J Microencapsulation 2003;20:211-25.

19. Puttipipatkhachorn S. Xanthan-alginate composite gel beads molecular interactions and in vitro characterization. Int J Pharm 2007;331:61-71.

20. Pongjanyakul T, Priprem A. Molecular interactions in alginate beads reinforced with sodium starch glycolate or magnesium silicate, and their physical characteristics. Int $\mathrm{J}$ Pharm 2005;293:51-62.

21. Gal A, Nussinovitch A. Hydrocolloid carriers with filler inclusion for diltiazem hydrochloride release. J Pharm Sci 2007;96:168-78.

22. Smrdel P, Bogataj M, Planinsek O, Mrhar A. Shape optimization and characterization of polysaccharide beads prepared by ionotropic gelation. J Microencapsul 2008;25:90-105.

23. Ferreira Almeida P. Cross-linked alginate-gelatin beads: a new matrix for controlled release of pindolol. J Controlled Release 2004; $97: 431-9$.

24. Lobenberg R, Amidon G. L, Modern bioavailability, bioequivalence and biopharmaceutics classification system. New scientific approaches to international regulatory standards. Eur J Pharm Biopharm 2000;50:3-12.

25. Park BJ. Current trends and future perspectives of solid dispersions containing poorly water-soluble drugs. Eur J Pharm Biopharm 2013;85:799-813.
26. Portincasa P, Mendez-Sanchez N, Wang H, Uribe, Wang DQ. Effect of ezetimibe on the prevention and dissolution of cholesterol gallstones. Gastro Enterol 2008;134:2101-10.

27. Garg R, Gupta GD. Progress in controlled gastro retentive delivery systems. Trop J Pharm Res 2008;7:1055-66.

28. Sung-Joo Hwang, Gye Ju Rhee, Ki Myung lee, Kyoug-Hee Oh, Chong-Kook Kim. Release characteristics of Ibuprofen from excipients-loaded alginate gel beads. Int J Pharm $2005 ; 116: 125-8$

29. Nimmi I, Chowdhury JA, Dulal MM, Reza MS. Effects of electrolytes on diclofenac sodium from agarose beads. Dhaka Univ J Pharm Sci 2005;4:117-20.

30. Pornsak Sriamornsak, Kanokporn Burapapadh, Satit Puttipipatkhachorn, Jurairat Nunthanid. Effect of acidic medium on selling and release behaviours of chitosan reinforced calcium pectinate gel beads. Sci-Tech J 2008;2:37-44.

31. Forizs E, Patrut A, Cozar IB, David L. Synthesis, structure elucidation of palladium complexes with theophylline. Acad Romana Roum Chim 2010;55:697-704.

32. Patel R, Bhimani D, Patel J, Patel D. Solid-state characterization and dissolution properties of ezetimibe-cyclo dextrin inclusion complexes. J Inclusion Phenom Macrocyclic Chem 2008;60:241-51.

33. Sancheti PP, Karekar P, VM Vyas VM, Shah M, Pore YV. Preparation and physicochemical characterization of surfactant based solid dispersions of ezetimibe. Pharmazie 2009;64:227-31.

34. Ostberg T, Vesterhus L, Graffner C. Calcium alginate matrices for oral multiple unit administration: II. Effect of process and formulation factors on matrix properties. Int J Pharm 1993;97:183-93.

35. Smrdel P, Bogataj M, Podlogar F, Planinsek O, Zajc N, Mazaj M, et al. Characterization of calcium alginate beads containing structurally similar drugs. Drug Dev Ind Pharm 2006;32:623-33.

36. Nunthanid J, Sriamornsak P. Calcium alginate gel beads for controlled release drug delivery: II. Effect of formulation and processing variables on drug release. J Microencapsul 1999:16:303-13.

37. Aslani P, Kennedy RA. Studies on diffusion in alginate gels. I. Effect of cross-linking with calcium or zinc ions on the diffusion of acetaminophen. J Controlled Release 1996;42:75-82.

38. Sriamornsak P. Investigation of pectin as a carrier for oral delivery of proteins using calcium pectinate gel beads. Int J Pharm 1998;169:213-20.

39. Larson I, Dass CR, Choong PF, Dunstan DE. A chitosan dipotassium orthophosphate hydrogel for the delivery of doxorubicin in the treatment of osteosarcoma. Biomaterials 2009;2:213-20.

40. Nayak AK, Hasnain MS, Beg S, Alam M. Mucoadhesive beads of gliclazide: Design, development and evaluation. Sci Asia 2010;36:319-25.

41. Nutan MTH, Soliman MS, Taha EI, Khan MA. Optimisation and characterization of controlled release multi-particulate beads coated with starch acetate. Int J Pharm 2005;294:89-101.

42. Takka, Gombotz WR, Wee SF. Protein releases from alginate matrices. Adv Drug Delivery Rev 1998;31:267-85.

43. Korsmeyer RW, Gurny R, Doelker E, Buri P, Peppas NA. Mechanisms of solute release from porous hydrophilic polymers. Int J Pharm 1983;15:25-35.

44. Takka S, Ocak O, Acarturk OHF. Formulation and investigation of nicardipine $\mathrm{HCl}$-alginate gel beads with factorial designbased studies. Eur J Pharm Sci 1998;6:241-6.

45. Ostberg T. Thesis, University of Oslo, Oslo, Norway; 1994.

46. Gaserod 0. Thesis, Norwegian University of Science and Technology, Trondheim, Norway; 1998.

47. Sakhare SS, Yadav AV, Jadhav PD. Design, development and characterization of mucoadhesive gastro spheres of carvedilol. Int J Appl Pharm 2016;8:37-42.

\section{How to cite this article}

- KV Ramana Reddy, MV Nagabhushanam. Process and parameters affecting drug release performance of prepared cross-linked alginate hydrogel beads for ezetimibe. Int J Pharm Pharm Sci 2017;9(2):254-262. 